\title{
What makes a galaxy radio-loud?
}

\author{
R. A. Ortega-Minakata ${ }^{1}$, J. P. Torres-Papaqui ${ }^{1}$, H. Andernach ${ }^{1}$, \\ R. Coziol $^{1}$, J. M. Islas-Islas ${ }^{1}$, I. Plauchu-Frayn ${ }^{2}$, D. M. Neri-Larios ${ }^{1}$ \\ and M. del C. Rojas-Granados ${ }^{3}$ \\ ${ }^{1}$ Departamento de Astronomía, Universidad de Guanajuato, C.P. 36000, Guanajuato, Mexico \\ ${ }^{2}$ Instituto de Astrofísica de Andalucía (CSIC), E-18008, Granada, Spain \\ ${ }^{3}$ División de Ingenierías, Universidad de Guanajuato, C.P. 36885, Salamanca, Mexico \\ email: rene@astro.ugto.mx
}

\begin{abstract}
We compare the Spectral Energy Distribution (SED) of radio-loud and radio-quiet AGNs in three different samples observed with SDSS: radio-loud AGNs (RLAGNs), Low Luminosity AGNs (LLAGNs) and AGNs in isolated galaxies (IG-AGNs). All these galaxies have similar optical spectral characteristics. The median SED of the RLAGNs is consistent with the characteristic SED of quasars, while that of the LLAGNs and IG-AGNs are consistent with the SED of LINERs, with a lower luminosity in the IG-AGNs than in the LLAGNs. We infer the masses of the black holes (BHs) from the bulge masses. These increase from the IG-AGNs to the LLAGNs and are highest for the RLAGNs. All these AGNs show accretion rates near or slightly below $10 \%$ of the Eddington limit, the differences in luminosity being solely due to different $\mathrm{BH}$ masses. Our results suggests there are two types of AGNs, radio quiet and radio loud, differing only by the mass of their bulges or BHs.
\end{abstract}

Keywords. galaxies: active, galaxies: evolution, galaxies: fundamental parameters, galaxies: statistics, radio continuum: galaxies

\section{Introduction}

The Spectral Energy Distribution (SED) of galaxies is a tool that could allow to make a physical and possibly evolutionary connection between galaxies showing different levels of AGN activity. In this study, we compare the SEDs of radio-loud and radio-quiet AGNs in three different samples with the typical SEDs of QSOs and quasars. Our samples are composed of SDSS galaxies which are Radio Loud (RLAGNs) with extended radio structures (Lin et al. 2010), Low Luminosity AGNs (LLAGNs), which turn out to reside mostly in groups and clusters (Torres-Papaqui et al. 2011), and AGNs in isolated galaxies (IG-AGNs, Coziol et al. 2011). The SEDs are based on radio flux densities from NVSS (1.4 GHz), FIR magnitudes from IRAS $(100,60,25,12 \mu \mathrm{m})$ and optical fluxes from SDSS $(5100 \AA)$. Except for a few detections with Chandra $(\sim 5 \mathrm{keV})$ in IG-AGNs $(5$ of 25 radio-detected IG-AGNs), no X-rays were found for the other AGNs. We used the stellar population synthesis code STARLIGHT to subtract a template from which we deduce the bulge mass of the galaxies.

\section{Sample Description and Results}

The RLAGNs and LLAGNs in our sample are mostly early-type galaxies in groups or clusters. Very few (142 of 4197) LLAGNs are detected in radio (NVSS at $1.4 \mathrm{GHz}$ ). Despite the difference in radio emission the RLAGNs and LLAGNs show similar spectra in the optical: both are narrow-line emission galaxies, frequently with some emission 

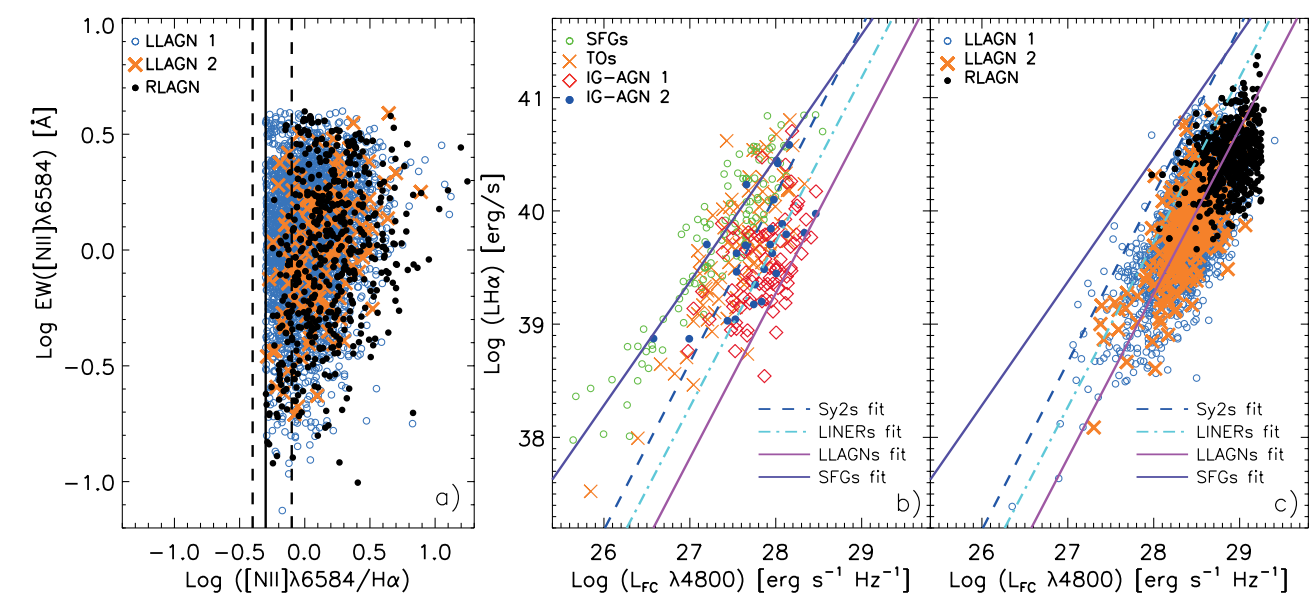

Figure 1. a) NII diagnostic diagram used to identify the LLAGNs and RLAGNs; b) and c) relations between the continuum luminosity at $4800 \AA$ and the $\mathrm{H} \alpha$ luminosity for the AGNs in IG-AGNs (b) and the LLAGNs (c). For comparison, in (b) and (c) we show power law fits for Sy2, LINERs, LLAGNs, and Star Forming Galaxies (SFGs), based on a total of $\sim 3 \times 10^{5}$ SDSS galaxies (Torres-Papaqui et al. 2011).

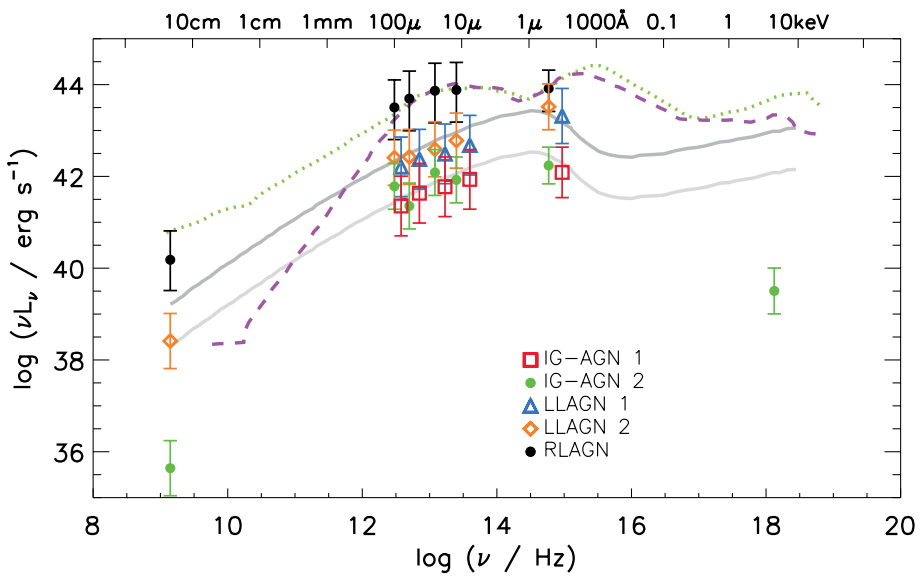

Figure 2. The SEDs of the different AGNs in our sample are compared with characteristic SEDs for quasars (dotted curve), QSOs (dashed curve) and LINERs (solid curves; one each for the LLAGNs and IG-AGNs). The symbols with error bars represent the median and quartiles. The data points for the IG-AGN 1 and LLAGN 1 samples were shifted slightly to the blue for clarity sake.

lines missing ([OIII $] \lambda 5007$ and/or $\mathrm{H} \beta$ ). The NII diagnostic diagram, Fig. 1a, was used to determine the nature of their activity (Torres-Papaqui et al. 2011). Galaxies with $\log [\mathrm{NII}] / \mathrm{H} \alpha>-0.3$ and $\log \mathrm{EW}[\mathrm{NII}]<0.6$ are classified as LLAGNs.

The IG-AGNs are mostly spiral galaxies with intense narrow emission lines. A standard diagnostic diagram was used to identify 104 AGNs among the 292 IGs. Only 25 of these were detected in NVSS. In Fig. 1b we show that both the radio-undetected (IG-AGN 1) and radio-detected (IG-AGN 2) IG-AGNs are mostly LINERs. In Fig. 1c we show that the RLAGNs are generally more luminous than both the radio-undetected (LLAGN 1) and radio-detected (LLAGN 2) LLAGNs.

In Fig. 2 we show the median SEDs of the AGNs in our sample, as compared with characteristic SEDs for quasars, QSOs and LINERs (Elvis et al. 1994; Younes et al. 2010). The SEDs of the quasars and QSOs were scaled down in luminosity to fit the optical data for the RLAGNs. The LINER SED was scaled up in luminosity to fit the optical and FIR data for the LLAGNs and the IG-AGNs. The observed radio power 

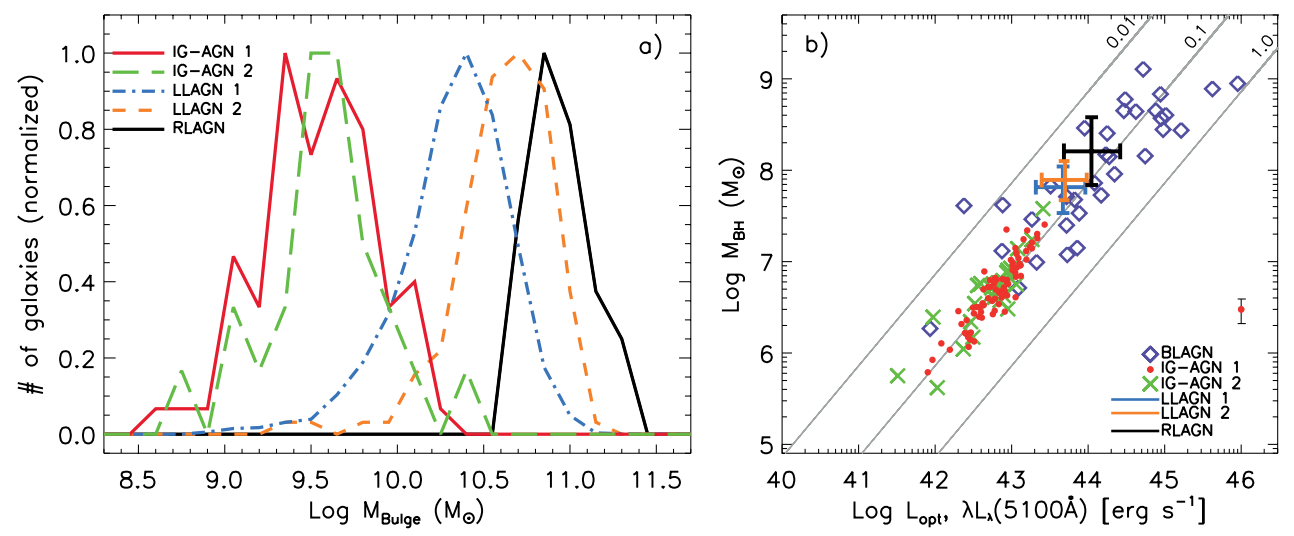

Figure 3. a) Normalized histograms for the bulge masses; b) relation between continuum luminosity at $5100 \AA$ and black hole mass as deduced from the mass of the bulge. The data for broad line AGNs (BLAGNs) are from Peterson et al. (2005).

is generally lower than expected from the characteristic SEDs and models. The few Xray detections with Chandra of galaxies in the IG-AGN 2 sample also fall much below expectation.

In Fig. 3a we find that the mass of the bulge decreases in the following order: RLAGNs, LLAGN 2, LLAGN 1, IG-AGN 2 and IG-AGN 1. In each sample, the radio-loud galaxies always have the more massive bulges. In Fig. 3b we show that all the black holes $(\mathrm{BHs})$ accrete at rates near or slightly below $10 \%$ of the Eddington limit. The differences in luminosity seem solely due to different $\mathrm{BH}$ masses, inferred from the relation by Häring \& Rix (2004).

\section{Discussion and Conclusions}

The similarity of the optical spectral characteristics of RLAGNs and LLAGNs suggests they have a common nature, implying they are intrinsically two types of AGNs, radio loud and radio quiet. The median SEDs of LLAGNs and IG-AGNs are consistent with scaled-up versions of the characteristic SED of LINERs, while that of RLAGNs is consistent with a scaled-down SED of quasars. The difference in bulge mass between radio-loud and radio-quiet AGNs suggests the central $\mathrm{BH}$ mass to be higher in radio-loud AGNs. The fact that the IG-AGNs, which have formed and evolved in isolation, have smaller BH masses, suggests that the environment of a galaxy may not only determine its morphology but also its AGN type.

J.P. T-P acknowledges support grants by PROMEP (103.5-10-4684), and DAIP-UGto (65/11). I. P-F acknowledges postdoctoral fellowship \#145727 by CONACyT, Mexico.

\section{References}

Coziol, R., Torres-Papaqui J. P., Plauchu-Frayn, I., et al. 2011, Rev. Mexicana AyA, 47, 361

Elvis, M., Wilkes, B. J., McDowell, J. C., et al. 1994, ApJS, 95, 1

Häring, N. \& Rix, H-W. 2004, ApJ, 604, L89

Lin, Y.-T., Shen, Y., Strauss, M. A., Richards, G. T., \& Lunnan, R. 2010, ApJ, 123, 1119

Peterson, B. M., Bentz, M. C., Desroches, L.-B., et al. 2005, ApJ, 632, 799

Torres-Papaqui, J. P., Coziol, R., Andernach, H., et al. 2011, MNRAS, submitted

Younes, G., Porquet, D., Sabra, B., et al. 2010, A\&A, 517, A33 